the poles. Calculations by S. Madronich (National Center for Atmospheric Research, Boulder) predict that for midand low-latitudes, fluxes of biologically active UVB $(290-320 \mathrm{~nm})$ at the surface will change by -5 to +10 per cent by the year 2060, depending both on the model and the assumed course of ozone depletion. For southern polar regions, large increases in springtime ultraviolet are expected (as much as a factor of ten in the year 2060). But because the average UVB irradiance is much higher at equatorial latitudes than at the poles, a percentage increase in UVB irradiance in the tropics corresponds to many more additional photons than the same increase at high latitudes.

Increases in solar ultraviolet radiation seem certain to produce substantial changes in the composition of the atmosphere as a consequence of the absorption of the radiation by gaseous molecules. Calculations by A.M. Thompson (NASA/ Goddard Space Flight Center) indicate that the oxidizing power of the lower atmosphere will be much increased and that the atmospheric content of many trace gases scavenged by oxidizers will decrease. Cloud effects pose large uncertainties, however, because soluble gasphase species can interact with clouds (and aid in their formation) in ways not well understood, and because most atmospheric chemical models have yet to treat cloud effects.

P. Crutzen (Max-Planck-Institute for Chemistry) reported recent calculations in which cloud chemistry was added to a global two-dimensional model. Although clouds occupy only about 15 per cent of the volume of the lower troposphere and liquid water occupies only $10^{-7}$ to $10^{-6}$ of a cloud's volume, the inclusion of clouds in the model greatly decreased the calculated concentrations of ozone, hydroxyl radical and nitrogen oxides, thereby affecting the budgets of many other trace gases (P. Crutzen and J. Lelieveld Nature, in the press).

Within condensed phases, the results of increased ultraviolet are also likely to be dramatic. A few years ago, there was little evidence for the existence of reactive chemical transients in environmental waters. Recently, however, several studies using carefully designed trapping-agent molecules have established the presence of such species as excited states, singlet molecular oxygen, solvated electrons, hydroxyl radicals, superoxide radicals, carbon-centred radicals and hydrogen peroxide. All are generated by solar ultraviolet radiation. Hydrogen peroxide is the longest lived of these transients and its widespread occurrence in the upper ocean and freshwaters provides an important marker of the influence of solar ultraviolet radiation in aquatic environments.

The biological effects, on both plants and animals, of increased ultraviolet radiation can be assessed by species only if each $A_{k}(\lambda)$, the appropriate species action spectrum, has been determined. Useable action spectra are, however, rare. $T$. Coohill (Western Kentucky University) highlighted some of the problems: uncertainty in the spectral purity of the incident radiation; difficult dosimetry determinations; the extent of biological repair before assay; the physical state of the target molecules; and response in vivo versus response in vitro. Hence, assessments of the effects of changing ultraviolet radiation are, at present, much more likely to be qualitative than quantitative.

In one of the workshop's more provocative presentations, F.M.M. Morel (Massachusetts Institute of Technology) argued that one could logically defend any of the following positions. Increased ultraviolet radiation will: promote a dramatic increase in new primary biotic productivity; practically destroy biotic productivity; or have no discernible effect on biotic productivity. His claim can be made because the quantitative information to determine the dominant effects is generally unavailable and because different factors seem likely to dominate at different places, times and seasons.

Morel's first position was supported by several participants who pointed out that the bioproductivity of the oceans often seems to be limited by the availability of trace nutrients such as iron, copper and manganese. Photochemical reactions involving bound forms of these elements increase their availability. Hence, regions of the oceans that are now nutrientlimited may benefit from increased ultraviolet radiation.

The converse position - that the increased damage to DNA will overwhelm any benefits in nutrient supply - was supported by several reports that UVB radiation markedly reduces growth in some species of marine phytoplankton. The biological impact is related to the number and efficiency of DNA repair systems in organisms. Field studies from Palmer Station, Antarctica (D. Karentz, University of California, San Francisco) demonstrate that there are enormous interspecies differences in sensitivity to ultraviolet.

Could increased ultraviolet fluxes have only little effect on biotic productivity? T. Mill (SRI International) argued that concentrations of marine and freshwater reactive chemical transients are too low and their lifetimes too short to have major adverse effects on the biota. It is also worth considering that marine phytoplankton already cope with reactive photoproducts and trace-element depletion.

T. E. Graedel is at AT\&T Bell Laboratories, Murray Hill, New Jersey 07974, USA.

\section{Air of longevity}

ONE of the appealing hazards of air travel is the ease with which you can get drunk. The reduced pressure and oxygen content of the cabin slows the metabolic elimination of alcohol, giving two drinks almost the effect of three. Daedalus therefore began to design a low-pressure pub. Drinkers would have to enter by an air-lock, but could then get drunk more cheaply, and stay that way longer. Conversely, increased pressure should speed up alcohol metabolism. A high-pressure bedroom, for example, might enable the alcoholic to burn off his entire hangover overnight, and greet the morning after with a clear head.

Daedalus is now extending this whole principle. He points out that if fed on restricted diets, nearly all animals mature more slowly and live longer than usual. Every creature studied seems to step through its whole 'life programme', from growth and sexual maturity to senescence and death, by the ticking of its metabolic clock. Animals raised in low pressures should therefore have longer, slower biological careers. If the principle works with man as well, then by spending your whole life in a cruising aircraft you might live to be a hundred. Or perhaps it would only seem like that.

Low pressure may even space out the minor milestones of life. The irregular menstrual cycle of many airline stewardesses has been attributed to their chaotic shuttling between time zones. Daedalus suspects instead that they 'age' more slowly in the air, and get out of step with groundlevel time.

So DREADCO's physiologists are setting up a variable-metabolism unit. To avoid the problems of pressurized construction, Daedalus contemplated slowing the oxygen metabolism of the inmates by introducing a little carbon monoxide into the air of the building. But wiser counsels prevailed, and instead the oxygen concentration of its air will be varied directly. Daedalus expects that his under-oxygenated volunteers will lose appetite and need only two meals a day. To study ageing itself, he is appealing for middle-aged identical twins, one to live in the low-oxygen laboratory and one outside while microscopic examination compares the rate at which grey hairs appear on their scalps.

The effects of oxygen-enriched air should also be interesting. Metabolism may speed up sharply, so that obese volunteers are swiftly 'burnt down' to normal weight. Animals may grow faster: an oxygenated poultry house, for example, might hurry chickens to earlier maturity. It would be hard to extend such atmospheric modifications to free-range life, whether in chickens or man; though an oxygen boost on the air conditioning might be welcome in many crisis-prone offices.
David Jones 